\title{
Some Magnetized Bianchi Type-III Massive String Cosmological Models in General Relativity
}

\author{
R. D. Upadhaya ${ }^{a *}$ and Shuchi Dave ${ }^{b}$ \\ ${ }^{a}$ Department of Mathematics Govt. P. G. College, Ajmer, India \\ ${ }^{b}$ Department of Mathematics, University of Rajasthan, Jaipur-302004, India
}

(Received on 3 September, 2008)

\begin{abstract}
Some Bianchi type III cosmological models with magnetic field for massive string are investigated. $\mathrm{F}_{12}$ is only the non-vanishing component of electromagnetic field tensor $F_{i j}$. To get a determinate solution. we have assumed that the expansion $(\theta)$ in the model is proportional to the shear $(\sigma)$. This leads to $C=A^{n}$ where $A$ and $C$ are functions of time alone. A particular solution for $n=1$ is discussed. The physical implications of the models, are also discussed.
\end{abstract}

Keywords: Cosmic massive string; Bianchi Type III, Magnetized

\section{INTRODUCTION}

It is still a challenging problem before us to know the exact physical situation at very early stages of the formation of our universe. The string theory is a useful concept before the creation of the particle in the universe. The string are nothing but the important topological stable defects due to the phase transition that occurs as the temperature lower below some critical temperature at the very early stages of the universe. The present day configuration of the universe are not contradicted by the large scale network of strings in the early universe. Moreover, the galaxy formation can be explained by the density fluctuations of the vacuum strings.

The general relativistic formalism of cosmic strings, are given by Letelier [1,2] and Stachel [3]. They have given the energy-momentum tensor for string distribution in the form

$$
T_{i j}=\rho u_{i} u_{j}-\lambda w_{i} w_{j}
$$

where

$$
u^{i} u_{i}=-w^{i} w_{i}=-1 \quad \text { and } \quad u^{i} w_{i}=0
$$

where $\rho$ is the energy density for a cloud of strings with particles attached to them, $\lambda$ the string tension density. The unit time like vector $u^{i}$ is the flow vector and the unit space like vector $w^{i}$ specifies the direction of the strings. The particle density associated with the configuration is given by

$$
\rho_{p}=\rho-\lambda
$$

Using these concepts, a number of general relativistic exact solutions were investigated, Krori et al [4] investigated Bianchi type II, VI $\mathrm{V}_{0}$, VIII and IX string cosmological models. A class of cosmological solutions of massive strings has been obtained by Chakraborty [5] for Bianchi type $\mathrm{VI}_{0}$ space time. Roy and Banerjee [6] have investigated some LRS Bianchi

*E-mail: dutt13@gmail.com type II string cosmological models which represent geometrical and massive strings. Banerjee et al [7] investigated some cosmological solutions of massive strings for Bianchi type I space-time in presence and absence of magnetic field. Tikekar and Patel [8] obtained some exact solutions of massive string of Bianchi type III space time in presence of magnetic field. They have also discussed some string solutions of Bianchi type III space time in absence of magnetic field. Lidsey et al [9] have discussed the aspects of super string cosmology with the cosmological implications of duality symmetries. Bali et al. [10-14] have a investigated Bianchi Types I, V, IX string cosmological models in General Relativity. Wang [15-18] has investigated and discussed some cosmological models and their physical implications in some Bianchi Type space-times. Melvin [19] in his cosmological solution for dust and electromagnetic field, has suggested that the presence of magnetic field is not as unrealistic as it appears to be because during the evolution of the universe, matter was in a highly ionized state, smoothly coupled with the field subsequently forms neutral matter due to universe expansion. Very recently Bali and Pradhan [20] have established a formalism for studying the new integrability of Bianchi Type III massive strings cosmological models in General Relativity.

In this paper, we have investigated Bianchi type III massive string cosmological models in presence of magnetic field. The string solution for general values of $n$ and $n=1$ are investigated. The physical implications of the models are also discussed. The behaviour of the models in presence and absence of magnetic field are also discussed.

\section{THE METRIC AND FIELD EQUATIONS}

We consider the Bianchi III space-time in the form

$$
d s^{2}=-d t^{2}+A^{2} d x^{2}+B^{2} e^{-2 \alpha x} d y^{2}+C^{2} d z^{2}
$$

where $\alpha$ is a constant and $A, B$ and $C$ are function of $t$ only.

We assume that the coordinate to be commoving so that

$$
u^{1}=0=u^{2}=u^{3} \quad \text { and } \quad u^{4}=1
$$


The energy momentum tensor for a cloud of string dust with magnetic field along the $z$-direction of the string, given by

$$
T_{i k}=\rho u_{i} u_{k}-\lambda w_{i} w_{k}+\frac{1}{4 \pi}\left[g^{\ell m} F_{i \ell} F_{k m}-\frac{1}{4} g_{i k} F_{\ell m} F^{\ell m}\right]
$$

where $u_{i}$ and $w_{i}$ satisfy conditions (1.2) is considered as the source term in the Einstein field equation

$$
R_{i k}-\frac{1}{2} R g_{i k}=-8 \pi T_{i k}
$$

In a comoving system, we have

$$
u^{i}=(0,0,0,1), \quad w^{i}=\left(0,0, \frac{1}{C}, 0\right)
$$

The electromagnetic field tensor $F_{i k}$ has only the non-zero component $F_{12}$ because the magnetic fields is assumed to be along the $z$-direction. Subsequently Maxwell equation

$$
F_{i k, \ell}+F_{k \ell, i}+F_{\ell i, k}=0 \quad \text { and } \quad\left[F^{i k}(-g)^{1 / 2}\right]_{, k}=0
$$

lead to

$$
F_{12}=K e^{-\alpha x}
$$

where $K$ is a constant so the magnetic field depends upon the space coordinate $x$ only. From (2.3), (2.5) and (2.6), it follows that $F_{14}=0$. The field equations (2.4) impose the requirement

$$
R_{14}=\alpha\left[\frac{\dot{A}}{A}-\frac{\dot{B}}{B}\right]=0
$$

Here and in what follows a dot denotes the derivative with respect to $t$. We have two cases $\alpha=0$ and $A=B$. When $\alpha=0$, the metric (2.1) degenerates into Bianchi type I considered by Banerjee et al [1990]. As we wish to consider space times with Bianchi III symmetry, we assume that $\alpha$ is non-zero and $A=B$ The field equation (2.4) lead to the following system of equations

$$
\begin{gathered}
8 \pi \rho=\frac{2 \dot{A} \dot{C}}{A C}+\frac{\dot{A}^{2}}{A^{2}}-\frac{\alpha^{2}}{A^{2}}-\frac{K^{2}}{A^{4}} \\
8 \pi \lambda=\frac{2 \ddot{A}}{A}+\frac{\dot{A}^{2}}{A^{2}}-\frac{\alpha^{2}}{A^{2}}-\frac{K^{2}}{A^{4}} \\
\frac{\ddot{A}}{A}+\frac{\ddot{C}}{C}+\frac{\dot{A} \dot{C}}{A C}+\frac{K^{2}}{A^{4}}=0
\end{gathered}
$$

where $\rho_{p}=$ Particle density $=\rho-\lambda$.

Thus the particle density $\rho_{p}$ is given by

$$
8 \pi \rho_{p}=-\frac{2 \ddot{A}}{A}+\frac{2 \dot{A} \dot{C}}{A C}
$$

in accordance with (1.3).

The velocity field $u_{i}$ is specified by (2.5) is irrotational. The expansion scalar $\theta$ and the shear scalar $\sigma^{2}$ are respectively found to have the following expressions

$$
\begin{gathered}
\theta=u_{, i}^{i}=\frac{2 \dot{A}}{A}+\frac{\dot{C}}{C} \\
\sigma^{2}=\frac{1}{2} \sigma_{i k} \sigma^{i k}=\frac{1}{3}\left[\frac{\dot{C}}{C}-\frac{\dot{A}}{A}\right]^{2}
\end{gathered}
$$

\section{SOLUTION OF THE FIELD EQUATIONS}

The field equations (1.2) are a system of three equations (28 -2.10) with four unknown parameters $\rho, \lambda, A$ and $C$. One additional constraint relating these parameters is required to obtain explicit solutions of the system. Referring to Thorne [21], observations of the velocity redshift relation for extragalatic sources suggest that Hubble expansion of the universe is isotropic within $30 \%$ range approximately $[22,23]$ and redshift studies place the limit $\frac{\sigma}{H} \leq 0.30$ where $\sigma$ is shear and $H$ the Hubble constant. Following Bali and Jain [24], we assume that the expansion $(\theta)$ is proportional to the shear $(\sigma)$ which is physically conditions. This condition leads to

$$
C=A^{n}
$$

where $n$ is a constant.

From equation (3.1) in equation (2.10), we get

$$
\frac{\ddot{A}}{A}+k \frac{\dot{A}^{2}}{A^{2}}=-\frac{\beta}{A^{4}}
$$

where

$$
k=\frac{n^{2}}{n+1}
$$

and

$$
\beta=\frac{K^{2}}{n+1}
$$

Now put $\dot{A}=f(A)$ in equation (3.2), we get

$$
\frac{d}{d A}\left(f^{2}\right)+\frac{2 k}{A} f^{2}=-\frac{2 \beta}{A^{3}}
$$

Further, equation (3.5) leads to

$$
f^{2}=\left(\frac{d A}{d t}\right)^{2}=L A^{-\frac{2 n^{2}}{n+1}}-\frac{K^{2} A^{-2}}{n^{2}-n-1}
$$


Thus the metric (2.1) reduces to the form

$$
\begin{aligned}
& d s^{2}=-\left(\frac{d t}{d A}\right)^{2} d A^{2}+A^{2} d x^{2}+A^{2} e^{-2 \alpha x} d y^{2}+A^{2 n} d z^{2} \\
& =-\frac{d T^{2}}{L T^{-2 n^{2} / n+1}-\frac{K^{2} T^{-2}}{n^{2}-n-1}}+T^{2}\left(d X^{2}+e^{-2 \alpha X} d Y^{2}\right)+T^{2 n} d Z^{2}
\end{aligned}
$$

where $A=T, x=X, y=Y, z=Z$.

In the absence of magnetic field i.e. when $K \rightarrow 0$ then the metric (3.8) reduces to the form

$$
d s^{2}=-\frac{d T^{2}}{L T^{-2 n^{2} / n+1}}+T^{2}\left(d X^{2}+e^{-2 \alpha X} d Y^{2}\right)+T^{2 n} d Z^{2}
$$

\subsection{SOME PHYSICAL AND GEOMETRICAL FEATURES}

The rest energy density $(\rho)$ and string tension density $(\lambda)$ for the model (3.7) are given by

$$
\begin{aligned}
& \rho=\frac{1}{8 \pi}\left[\frac{-K^{2}(2 n+1)}{\left(n^{2}-n-1\right) T^{4}}+\frac{(2 n+1) L}{T^{2} \frac{\left(n^{2}+n+1\right)}{n+1}}-\frac{\alpha^{2}}{T^{2}}-\frac{K^{2}}{T^{4}}\right] \\
& \lambda=\frac{1}{8 \pi}\left[\frac{K^{2}}{\left(n^{2}-n-1\right) T^{4}}-\frac{\left(2 n^{2}-1\right) L}{(n+1) T^{2} \frac{\left(n^{2}+n+1\right)}{n+1}}-\frac{\alpha^{2}}{T^{2}}-\frac{K^{2}}{T^{4}}\right]
\end{aligned}
$$

The particle density $\rho_{p}$ is given by

$$
\rho_{p}=\rho-\lambda=\frac{1}{8 \pi}\left[\frac{-2 K^{2}(n+1)}{\left(n^{2}-n-1\right) T^{4}}+\frac{n(4 n+3) L}{(n+1) T^{2} \frac{\left.n^{2}+n+1\right)}{n+1}}\right]
$$

The scalar of expansion $(\theta)$ and shear $(\sigma)$ for the model (3.7) are given by

$$
\begin{aligned}
& \theta=(n+2)\left[\frac{-K^{2}}{\left(n^{2}-n-1\right) T^{4}}+\frac{L}{T^{2} \frac{\left.n^{2}+n+1\right)}{n+1}}\right]^{1 / 2} \\
& \sigma^{2}=\frac{1}{3}(n-1)^{2}\left[\frac{-K^{2}}{\left(n^{2}-n-1\right) T^{4}}+\frac{L}{T^{2} \frac{\left.n^{2}+n+1\right)}{n+1}}\right]
\end{aligned}
$$

The energy condition $\rho \geq 0$ implies that

$$
\frac{(2 n+1) L}{T^{2} \frac{\left.n^{2}+n+1\right)}{n+1}} \geq \frac{\alpha^{2}}{T^{2}}+\frac{K^{2}(2 n+1)}{\left(n^{2}-n-1\right) T^{4}}+\frac{K^{4}}{T^{4}}
$$

The particle density and tension density of the cloud string vanish asymptotically in general if $n^{2}+n+1>0$. The expansion in the model stops when $n=-2$. The model starts expanding with a big bang at $T=0$ and the expansion in the model decreases as time increases if $n^{2}+n+1>0$. Since $\lim _{T \rightarrow \infty} \frac{\sigma}{\theta} \neq 0$. Hence the model does not approach isotropy for large values of $T$. The model (3.7) has singularity at $T=0$. The physical parameters $\rho, \lambda, \rho_{p}$ are infinite at the singularity $T=0$ and monotonically decreasing as $T \rightarrow \infty$. The energy density and expansion in the model decreases more rapidly in presence of magnetic field. In absence of magnetic field, the energy condition $\rho \geq 0$ lead to

$$
\frac{2 n^{2}}{T^{n+1}} \leq \frac{(2 n+1) L}{\alpha^{2}}
$$

In absence of magnetic field, the physical parameters $\rho, \lambda, \rho_{p}, \theta$ and $\sigma$ are given by

$$
\begin{gathered}
\rho=\frac{1}{8 \pi}\left[\frac{(2 n+1) L}{T^{2} \frac{\left(n^{2}+n+1\right)}{n+1}}-\frac{\alpha^{2}}{T^{2}}\right] \\
\lambda=-\frac{1}{8 \pi}\left[\frac{\left(2 n^{2}-1\right) L}{(n+1) T^{2} \frac{\left(n^{2}+n+1\right)}{n+1}}+\frac{\alpha^{2}}{T^{2}}\right] \\
\rho_{p}=\frac{1}{8 \pi} \frac{L n(4 n+3)}{T^{2} \frac{\left(n^{2}+n+1\right)}{n+1}} \\
\theta=\frac{(n+2) L^{1 / 2}}{T \frac{n^{2}+n+1}{n+1}} \\
\sigma^{2}=\frac{1}{3} \frac{(n-1)^{2} L}{T^{2} \frac{\left(n^{2}+n+1\right)}{n+1}}
\end{gathered}
$$

In absence of magnetic field, the particle density $\rho_{p}$ and tension density $\lambda$ vanish when $T \rightarrow \infty$. The expansion in the model decreases as time increases if $n^{2}+n+1>0$. However, if $n^{2}+n+1<0$ then the model starts expanding at $T=0$ and goes on expanding indefinitely. Since $\lim _{T \rightarrow \infty} \frac{\sigma}{\theta} \neq 0$. Then the model does not approach isotropy for large values of $T$.

\section{SPECIAL CASE}

When $n=1$ in equation (3.1), we get

$$
A=C
$$

Using equation (4.1) in equation (2.10), we get 
where

$$
\frac{2 \ddot{A}}{A}+\frac{\dot{A}^{2}}{A^{2}}+\frac{K^{2}}{A^{4}}
$$

We solve equation (4.2) by putting

$$
\dot{A}=f(A)
$$

so we get

$$
f^{2}=\frac{K^{2}+N A}{A^{2}}
$$

where $N$ is a constant

Further, we solve equation (4.4) by putting

$$
K^{2}+N A=\xi^{2}
$$

so we get

$$
\xi^{3}-3 K^{2} \xi-\left(\frac{3}{2} N^{2} t+3 M\right)=0
$$

where $M$ and $N$ are constants.

The equation (4.6) leads to

$$
\begin{aligned}
& \xi=\left[\frac{3}{2}\left(\frac{N^{2}}{2} t+M\right)+\frac{3}{2} \sqrt{\left(\frac{N^{2}}{2} t+M\right)^{2}-K^{6}}\right]^{1 / 3} \\
& +\left[\frac{3}{2}\left(\frac{N^{2}}{2} t+M\right)-\frac{3}{2} \sqrt{\left(\frac{N^{2}}{2} t+M\right)^{2}-K^{6}}\right]^{1 / 3}
\end{aligned}
$$

Using equation (4.5) in (4.7), we get

$$
A=\frac{\left[(a+b)^{1 / 3}+(a-b)^{1 / 3}\right]^{2}-K^{2}}{N}
$$

$$
a=\frac{3}{2}\left(\frac{N^{2}}{2} t+M\right)
$$

and

$$
b=\frac{3}{2} \sqrt{\left(\frac{N^{2}}{2} t+M\right)^{2}-K^{6}}
$$

Now metric (2.1) becomes

$$
d s^{2}=-d t^{2}+\frac{\left[\left\{(a+b)^{1 / 3}+(a-b)^{1 / 3}\right\}^{2}-K^{2}\right]}{N^{2}}
$$

where

$$
\left\{d x^{2}+e^{-2 \alpha x} d y^{2}+d z^{2}\right\}
$$

$$
a+b=\frac{3}{2}\left[\left(\frac{N^{2}}{2} t+M\right)+\sqrt{\left(\frac{N^{2}}{2} t+M\right)^{2}-K^{6}}\right]
$$

and

$$
a-b=\frac{3}{2}\left[\left(\frac{N^{2}}{2} t+M\right)-\sqrt{\left(\frac{N^{2}}{2} t+M\right)^{2}-K^{6}}\right]
$$

\subsection{PHYSICAL AND GEOMETRICAL FEATURES}

The rest energy density $(\rho)$ amd string tension density $(\lambda)$ for the model (4.11) are given by

$$
\begin{gathered}
8 \pi \rho=\frac{3 N^{4}\left[(a+b)^{2 / 3}-(a-b)^{2 / 3}\right]^{2}}{4 b^{2}\left\{\left[(a+b)^{1 / 3}+(a-b)^{1 / 3}\right]^{2}-K^{2}\right\}^{2}}-\frac{\alpha^{2} N^{2}}{\left\{\left[(a+b)^{1 / 3}+(a-b)^{1 / 3}\right]^{2}-K^{2}\right\}^{2}} \\
-\frac{K^{2} N^{4}}{\left\{\left[(a+b)^{1 / 3}+(a-b)^{1 / 3}\right]^{2}-K^{2}\right\}^{4}} \\
8 \pi \lambda=\frac{N^{4}}{4 b^{3}} \frac{\left[(a+b)^{2 / 3}(2 b-3 a)+(a-b)^{2 / 3}(2 b+3 a)\right]}{\left\{\left[(a+b)^{1 / 3}+(a-b)^{1 / 3}\right]^{2}-K^{2}\right\}}+\frac{N^{4}\left[(a+b)^{2 / 3}-(a-b)^{2 / 3}\right]^{2}}{4 b^{2}\left\{\left[(a+b)^{1 / 3}+(a-b)^{1 / 3}\right]^{2}-K^{2}\right\}^{2}} \\
-\frac{\alpha^{2} N^{2}}{\left\{\left[(a+b)^{1 / 3}+(a-b)^{1 / 3}\right]^{2}-K^{2}\right\}^{2}}-\frac{K^{2} N^{4}}{\left\{\left[(a+b)^{1 / 3}+(a-b)^{1 / 3}\right]^{2}-K^{2}\right\}^{4}}
\end{gathered}
$$


The particle density is given as

$$
\begin{gathered}
8 \pi \rho_{p}=8 \pi(\rho-\lambda)=-\frac{N^{4}}{4 b^{3}} \frac{\left[(a+b)^{2 / 3}(2 b-3 a)+(a-b)^{2 / 3}(2 b+3 a)\right]}{\left\{\left[(a+b)^{1 / 3}+(a-b)^{1 / 3}\right]^{2}-K^{2}\right\}} \\
+\frac{N^{4}\left[(a+b)^{2 / 3}-(a-b)^{2 / 3}\right]^{2}}{2 b^{2}\left\{\left[(a+b)^{1 / 3}+(a-b)^{1 / 3}\right]^{2}-K^{2}\right\}^{2}}
\end{gathered}
$$

where

$$
\begin{gathered}
a=\frac{3}{2}\left(\frac{N^{2}}{2} t+M\right) \\
b=\frac{3}{2} \sqrt{\left(\frac{N^{2}}{2} t+M\right)^{2}-K^{6}} \\
a+b=\frac{3}{2}\left[\left(\frac{N^{2}}{2} t+M\right)+\sqrt{\left(\frac{N^{2}}{2} t+M\right)^{2}-K^{6}}\right] \\
a-b=\frac{3}{2}\left[\left(\frac{N^{2}}{2} t+M\right)-\sqrt{\left(\frac{N^{2}}{2} t+M\right)^{2}-K^{6}}\right]
\end{gathered}
$$

The scalar of expansion $(\theta)$ and shear $(\sigma)$ for the metric (4.11) are given by

$$
\begin{gathered}
\theta=\frac{3 N^{2}}{2 b} \frac{\left\{(a+b)^{2 / 3}-(a-b)^{2 / 3}\right\}}{\left[\left\{(a+b)^{1 / 3}+(a-b)^{1 / 3}\right\}^{3}-K^{2}\right]} \\
\sigma^{2}=0
\end{gathered}
$$

The energy condition $\rho \geq 0$ leads to

$$
\begin{aligned}
& {\left[(a+b)^{2 / 3}-(a-b)^{2 / 3}\right] \geq \frac{4 b^{2} \alpha^{2}}{3 N^{2}}+} \\
& \frac{4 b^{2} K^{2}}{3\left[\left\{(a+b)^{1 / 3}+(a-b)^{1 / 3}\right\}^{2}-K^{2}\right]^{2}}
\end{aligned}
$$

The expansion in the model vanishes when $t=-\frac{2 M}{N^{2}}$ and when $t \rightarrow \infty$. Thus expansion in the model represents nonshearing and isotropic universe.
For $n=1$, in the absence of magnetic field, the energy density $(\rho)$, tension density $(\lambda)$ and particle density $\rho_{p}$, are given by

$$
\begin{gathered}
8 \pi \rho=\frac{N^{4}}{3 T^{2}}-\frac{\alpha^{2} N^{2}}{(3 T)^{4 / 3}} \\
8 \pi \lambda=\frac{N^{4}}{9 T^{2}}-\frac{N^{2}}{9 T^{2}}-\frac{\alpha^{2} N^{2}}{(3 T)^{4 / 3}} \\
8 \pi \rho_{p}=\frac{2 N^{4}}{9 T^{2}}+\frac{N^{2}}{9 T^{2}}
\end{gathered}
$$

The expansion $(\theta)$ and the shear $(\sigma)$ in the model are given by

$$
\theta=\frac{N^{2}}{T}
$$

and

$$
\sigma^{2}=0
$$

The energy condition $\rho \geq 0$ leads to

$$
T^{2 / 3} \leq \frac{3^{1 / 3} N^{2}}{\alpha^{2}}
$$

The model starts expanding with a big-bang $T=0$ and the expansion in the model decreases as time increases and the expansion in the model stops at $T=\infty$. The particle density and tension density of the cloud string vanish asymptotically in the model:

$$
d s^{2}=-4 / N^{4} d T^{2}+(3 T)^{4 / 3} / N^{2}\left\{d x^{2}+e^{-2 \alpha x} d y^{2}+d z^{2}\right\}
$$

It has singularity at $T=0$. The physical parameters $\rho, \lambda$, $\rho_{p}$ are infinite at the singularity $T=0$ and monotonically decreasing. 
[3] J. Stachel, Phys. Rev. D 21, 217 (1980).

[4] K. D. Krori, T. Chaudhary, C. R. Mahanta, and A. Mazumdar, Gen. Relativity \& Grav. 22, 123 (1990).

[5] S. Chakraborty, Ind. J. Pure and Appl. Phys. 29, 31 (1991).

[6] S. R. Roy and S. K. Banerji, Class Quantum Grav. 11, 1943 (1995).

[7] A. Banerjee, A. K. Sanyual, and S. Chakraborty, Pramana - J. Phys. 34, 1 (1990).

[8] R. Tikekar and L. K. Patel, Gen. Relativity \& Grav. 24, 397 (1992).

[9] J. E. Lidsey, D. Wands, and E. J. Copeland, Phys. Rep. 337, 343 (2000).

[10] R. Bali and S. Dave, Pramana - J. Phys. 56, 513 (2001).

[11] R. Bali and R. D. Upadhaya, Astrophysics and Space-Science 283, 97 (2003).

[12] R. Bali and S. Dave, Astrophysics and Space-Science 288, 503 (2003).
[13] R. Bali and D. K. Singh, Astrophysics and Space-Science 300 387 (2005).

[14] R. Bali and Anjali, Astrophysics and Space-Science 302, 201 (2006).

[15] X. X. Wang, Astrophysics and Space-Science 293, 933 (2004).

[16] X. X. Wang, Chin. Phys. Lett. 21, 1205 (2004).

[17] X. X. Wang, Chin. Phys. Lett. 22, 29 (2005).

[18] X. X. Wang, Chin. Phys. Lett. 23, 1702 (2006).

[19] M. A. Melvin, Ann. New York Acad. Sci. 262, 253 (1975).

[20] R. Bali and A. Pradhan, Chin. Phys. Lett. 24, 585 (2007).

[21] K. S. Thorne, Astrophys. J. 148, 51 (1967).

[22] R. Kanrowski and R. K. Sachs, J. Math. Phys. 7, 433 (1966).

[23] J. Kristian and R. K. Sachs, Astrophys. J. 143, 379 (1966).

[24] R. Bali and V. C. Jain, Astrophys. and Space-Science 262, 145 (1999). 